\section{VIVÊNCIAS DE TRABALHADORES DA SAÚDE: DIAGNÓSTICO E NOTIFICAÇÃO DE SÍFILIS NA ATENÇÃO BÁSICA}

\section{EXPERIENCES OF HEALTH WORKERS: DIAGNOSIS AND NOTIFICATION OF SYPHILIS IN BASIC CARE}

Jobe Lino Ferreira ${ }^{1, *} /$ Cleuma Sueli Santos Suto ${ }^{1} /$ Rita de Cássia Dias Nascimento $1 /$ Laura Emmanuela Lima Costa ${ }^{2}$ / Silvana Nunes Piva ${ }^{1}$ / Dejeane de Oliveira Silva ${ }^{3}$

\section{INTRODUÇÃO}

A sífilis é uma patologia infecciosa e contagiosa transmitida na relação sexual, por transfusão sanguínea e contatos íntimos, causada pela bactéria Treponema pallidum. Desde o século XV já era considerada uma das principais pragas mundiais, estando associada à doenças dermatológicas, como a Hanseníase, por apresentar sinais e sintomas relacionados a epiderme e a mucosas (SANTOS, et al., 2014)

De acordo o Ministério da Saúde (MS), a doença é de notificação compulsória e classifica-se em: sífilis adquirida, sífilis congênita (SC) e sífilis gestacional. A sífilis congênita se destaca frente as demais formas no contexto da saúde pública, devido aos desfechos graves que são produzidos na gestação e transmitidos à criança, fazendo-se necessário o rastreio precoce e respectiva notificação da sífilis adquirida e gestacional (DANTAS, et al., 2017).

Vale destacar, no entanto, que em pleno século XXI, as dificuldades para diagnóstico da sífilis, bem como a realização dos exames laboratoriais, podem estar relacionadas à carência de recursos tecnológicos complexos e às questões organizacionais, dentre outros motivos (LOPES, et al., 2016). Nesse sentido, as políticas do MS, no âmbito da atenção básica, com a proposta de intensificar as ações de combate à sífilis, dispõem sobre Testes Rápidos (TR) que favorecem a atuação direta na ampliação do acesso ao diagnóstico, possibilitando, assim, que pessoas acessem sua condição sorológica, realizem tratamento oportuno e diminuam a cadeia de transmissibilidade (MIZEVSKI, et al., 2017). \begin{abstract}
RESUMO
Objetivou-se identificar como profissionais de saúde lidam com o diagnóstico/notificação da sífilis e descrever as dificuldades e enfrentamentos vivenciados em seu manejo na atenção básica. Estudo qualitativo, descritivo-exploratório, tendo como colaboradores 50 profissionais/trabalhadores em saúde. Realizou-se entrevista semiestruturada, analisadas com auxílio do software Iramuteq baseado na análise fatorial de correspondência. Os achados deste estudo revelaram que a assistência prestada e as ações ofertadas, quanto ao diagnóstico e notificação da sífilis, se apresentavam fragilizada, desvelando deficiências na capacitação da equipe. Os resultados apontam para a necessidade de promoção de estratégias eficazes, como a do aconselhamento para atendimento às vulnerabilidades desses usuários e, indicam que intervenções políticas, gerenciais e de educação em serviço, direcionadas às equipes/serviços, careçam de ser implantadas, para um melhor enfrentamento da sífilis e promoção da saúde.
\end{abstract}

Palavras-chave: Sífilis. Serviços de Saúde. Gestantes. Atenção Básica.

\section{ABSTRACT}

The objective was to identify how health professionals deal with the diagnosis/notification of syphilis and to describe the difficulties and confrontations experienced in their management in primary care. Qualitative, descriptive, and exploratory study, with 50 professionals/health workers as collaborators. A semi-structured interview was performed, analyzed with the aid of Iramuteq software, based on correspondence factor analysis. The findings of this study revealed that the care provided, and the actions offered regarding the diagnosis and notification of syphilis are fragile, revealing deficiencies in the training of the team. The results point to the need to promote effective strategies, such as counseling to address the vulnerabilities of these users, and indicate that political, managerial and in-service education interventions, aimed at teams/services, need to be implemented, for a better coping with syphilis and health promotion.

Keywords: Syphilis. Health Services. Pregnant Women. Primary Care.

Submetido em: 4 de nov. 2019

Aceito em: 3 de set. 2020

${ }^{1}$ Universidade do Estado da Bahia - UNEB, Senhor do Bonfim, Bahia - Brasil.

${ }^{2}$ Universidade do Estado da Bahia - UNEB, Jacobina, Bahia - Brasil.

${ }^{3}$ Universidade Estadual de Santa Cruz - UESC, Ilhéus, Bahia - Brasil.

*E-mail para correspondência: jobe.uneb@gmail.com

Rev. ComCiência - dez. 2020, vol. 5, no. 7, p. 12-21/ doi: 10.36112/issn2595-1890.v5.i7.p12-21 
Contudo, os serviços de saúde são compostos por profissionais pouco preparados para a realização e entrega de resultados de exames ou para lidar com resultados positivos para sífilis, ocasionando, como consequência o diagnóstico tardio. Tais dificuldades impactam diretamente na estatística que, a cada ano, apresenta em média 12 milhões de pessoas no mundo infectadas por esta patologia e ranqueia a sífilis à condição de um grave problema de saúde pública (TIAGO, et al., 2017).

Nos últimos cinco anos, no Brasil e no estado da Bahia, houve crescimento significativo do número de casos de sífilis. Esse aumento pode ser atribuído, em partes, à ampliação da cobertura de testagem por meio de Testes Rápidos (TR) e/ou redução do uso do preservativo e resistência dos profissionais em administrar penicilina.

Vale salientar, que nos últimos anos, houve investimentos por parte do Estado para o aprimoramento do sistema de vigilância o que impactou diretamente no volume de casos notificados. A situação epidemiológica da sífilis no Brasil, no ano de 2018, registra 53.830 casos de sífilis em gestantes e 23.935 casos de sífilis congênita. Ressalta-se os óbitos decorrentes da sífilis congênita que somaram 206 casos e, o percentual de parceiros não tratados que foi maior que 50\% dentre os casos diagnosticados. (BRASIL, 2018; BRASIL, 2019)

Na Bahia, a situação epidemiológica da sífilis, entre janeiro de 2007 e junho de 2019, revelada através das notificações no Sistema Informação de Agravos e Notificação (SINAN) aponta 11.993 casos de sífilis congênita em menores de um ano de idade. No que tange a casos de sífilis em gestantes, os dados mostram 18.537 casos notifica- dos. Já os registros de sífilis adquirida (sífilis em adultos e sífilis não especificada), no período de janeiro de 2012 a 10 de junho de 2019, perfazem 46.308 notificações. No município de Senhor do Bonfim, foco deste estudo, os dados acompanham o perfil epidemiológico nacional e estadual e, entre os anos de 2007 a 2019, foram registrados 85 casos de sífilis em gestantes e 50 casos de sífilis congênita. Em relação à sífilis adquirida, as notificações neste período somam 272 casos. (BRASIL, 2019; BAHIA, 2019)

Diante do contexto em que a sífilis apresenta-se no cenário nacional, estadual e municipal, e das possíveis dificuldades em operacionalizar ações efetivas na atenção básica, a realização deste estudo encontra aporte ao questionar: Como serviços de saúde da atenção básica diagnosticam e/ou notificam a sífilis? Acreditando que o discurso de profissionais da atenção básica como médicos, enfermeiras, técnicos e auxiliares de enfermagem pudessem fornecer evidências específicas sobre os nexos entre as práticas de cuidado e o contexto vivenciado acerca da problemática da sífilis, delineou-se os seguintes objetivos: Identificar como profissionais de saúde lidam com o diagnóstico/notificação da sífilis e descrever as dificuldades e enfrentamentos vivenciados na atenção básica em seu 'manejo. Os dados aqui apresentados, constituem recorte de Trabalho de Conclusão de Curso.

Este estudo torna-se relevante para profissionais de saúde e saúde coletiva por facultar reflexões em torno da assistência ofertada à população e, quicá, preencherá lacunas existentes entre a teoria e a prática profissional.

\section{MÉTODO}

O estudo caracteriza-se como pesquisa de campo, de natureza básica, descritivo-exploratório e com abordagem qualitativa (MINAYO, 2016). Foi realizado no município de Senhor do Bonfim, localizado na região norte do Estado da Bahia-Brasil, tendo como lócus 12 unidades básicas de saúde (UBS). Foram abordados profissionais/trabalhadores em saúde, sendo: 05 (cinco) médicos, 12 (doze) enfermeiros, 06 (seis) odontólogos, 23 (vinte e três) técnicos de enfermagem e 04 (quatro) recepcionistas, totalizando uma amostra de 50 participantes, elegíveis conforme critérios de inclusão: estar trabalhando há pelo menos seis meses na UBS independentemente do tipo de vínculo. Como critério de exclusão: trabalhadores que atuam em UBS localizadas na zona rural do município. Os dados foram coletados no período de julho a outubro de 2018 pelo pesquisador principal.

As entrevistas para aplicação do instrumento foram previamente agendadas, aconteceram nos turnos da manhã e da tarde de acordo com o funcionamento das UBS e disponibilidade dos participantes. Inicialmente foi feita uma breve apresentação e exposição dos objetivos da pesquisa. Em seguida, procedeu-se a leitura das perguntas abertas e fechadas. As entrevistas foram gravadas em equipamento MP3, após consentimento prévio do participante, aquiescência e assinatura do Termo de Consentimento Livre e Esclarecido (TCLE). A duração média das entrevistas foi de 15 minutos.

Para organização das perguntas abertas, foi realizada a transcrição e a formatação dos textos usando o programa Microsoft Word e, em seguida, leituras exaustivas e confrontação com os áudios, compondo o corpus. No processo da análise utilizou-se o software Iramuteq (Interface de $\mathrm{R}$ pour les Analyses Multidimensionnelles de 
Textes et de Quationnaires), para melhor sistematizar os achados, uma vez que o mesmo permite proceder análises estatísticas sobre corpus textuais como: estatísticas textuais clássicas; pesquisa de especificidades a partir de segmentação definida do texto; Classificação Hierárquica Descendente (CHD); Análise Fatorial de Correspondência (AFC) (MENDES, et al., 2016).

Nesse estudo, o método de análise baseou-se na Análise Fatorial de Correspondência em que os 50 textos, advindos das questões abertas, foram classificados de acordo com seus respectivos vocábulos, a partir das análises realizadas pelo Iramuteq que delineou as Classes e suas relações. Assim, através da execução dos cálculos, o software forneceu resultados que possibilitam descrever as Classes (CAMARGO; JUSTO, 2013). O corpus textual proveniente da coleta de dados foi fragmentado em seis classes com um aproveitamento de $73,2 \%$ do material empírico.

A AFC apresenta o primeiro fator (F1) que explica $37,4 \%$ da inércia total e possui valor próprio de 0,098654; o segundo fator (F2) que explica $22,2 \%$ da inércia total, com valor próprio de 0,043241. O gráfico gerado pelo Iramuteq permitiu a apresentação e discussão das variáveis sexo, idade, tempo de atuação na UBS, formação e tempo de formação que compuseram cada uma das Classes.

Buscando contextualizar as palavras/termos presente no gráfico da AFC procedeu-se a inserção de expressões retiradas dos discursos dos entrevistados. Os excertos possibilitaram a identificação das ideias chave e maior compreensão dos termos dispostos na AFC. Tanto a figura gerada pelo software quanto os recortes de falas foram analisadas e interpretadas de acordo com a literatura vigente.

Para fins de atendimento aos princípios éticos na pesquisa houve aprovação pelo Comitê de Ética em Pesquisa, sob parecer número 2.590.142 e CAAE: 85912618.6.0000.0057, com base nas normas estabelecidas pela Resolução do Conselho Nacional de Saúde 466/12 que trata da pesquisa envolvendo seres humanos. A fim de preservar o anonimato as falas dos participantes foram identificadas com a letra correspondente à sua profissão/atividade, seguida no número atribuído de acordo com a ordem cronológica de participação, a exemplo: Enfermeira 01 (E1).

\section{RESULTADOS}

Os profissionais/trabalhadores que participaram do estudo em sua maioria eram mulheres $(72 \%)$, sendo 12 (doze) enfermeiras/os com idade entre 30-47 anos, 05 (cinco) médicos com idade entre 27-33 anos, seis (seis) odontólogos com idade entre 26-41 anos, 23 (vinte e três) técnicos de enfermagem com idade entre 23-62 anos e 04 (quatro) recepcionistas com idade entre 31-58 anos.

Com relação ao tempo de atuação desses profissionais nas UBS pesquisadas, há uma variação entre menos de um 01 (um) ano até mais de 05 (cinco) anos sendo, que a maioria (44\%) atua entre 01 (um) e 05 (cinco) anos. No que se refere à formação, destaca-se a ocorrência de 15 (quinze) participantes que possuíam curso de pós-graduação (especialização ou mestrado), dentre eles 11 (onze) são enfermeiros e 04 (quatro) odontólogos. As demais categorias profissionais que participaram não possuíam pósgraduação. $\mathrm{O}$ quadro 01 apresenta as variáveis tempo de atuação, formação e tempo de formação.

Quadro 1: Características sociodemográfica dos profissionais da saúde da atenção básica participantes da pesquisa, Senhor do Bonfim, 2019.

\begin{tabular}{|c|c|c|c|c|c|c|c|}
\hline \multicolumn{2}{|c|}{$\begin{array}{l}\text { Profissionais } \\
\text { da saúde }\end{array}$} & $\begin{array}{l}\text { Enfer- } \\
\text { mei } \\
\text { ros } \\
(\mathrm{n}=12)\end{array}$ & \begin{tabular}{|l} 
Médi- \\
cos \\
$(\mathrm{n}=05)$
\end{tabular} & $\begin{array}{l}\text { Odontó } \\
\text { logos } \\
(\mathrm{n}=06)\end{array}$ & $\begin{array}{l}\begin{array}{l}\text { Tec. de } \\
\text { enf. } \\
(\mathrm{n}=23)\end{array}\end{array}$ & $\begin{array}{l}\text { Recep- } \\
\text { cionis- } \\
\text { tas } \\
(\mathrm{n}=04)\end{array}$ & $\begin{array}{l}\text { Total } \\
(\mathrm{n}=50)\end{array}$ \\
\hline \multicolumn{2}{|c|}{$\begin{array}{l}\text { Percentual dos } \\
\text { profissionais } \\
\text { dentre as } \\
\text { categorias }(\%)\end{array}$} & 24 & 10 & 12 & 46 & 8 & 100 \\
\hline \multirow{3}{*}{ 造 } & $\begin{array}{c}\text { Até } 1 \\
\text { ano }\end{array}$ & 00 & 03 & 02 & 05 & 01 & 11 \\
\hline & $\begin{array}{l}1-5 \\
\text { anos }\end{array}$ & 09 & 02 & 03 & 06 & 02 & 22 \\
\hline & $>5$ anos & 03 & 00 & 01 & 12 & 01 & 17 \\
\hline \multirow{4}{*}{ 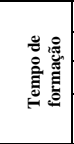 } & $<1$ ano & 00 & 00 & 00 & 00 & 00 & 0 \\
\hline & 1-3 anos & 00 & 03 & 01 & 01 & 00 & 05 \\
\hline & 4-9 anos & 06 & 01 & 01 & 04 & 00 & 12 \\
\hline & $\begin{array}{l}>=10 \\
\text { anos }\end{array}$ & 06 & 01 & 04 & 18 & 04 & 33 \\
\hline \multirow{4}{*}{ 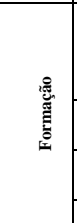 } & $\begin{array}{c}\text { Não } \\
\text { possui } \\
\text { especia- } \\
\text { lização } \\
\end{array}$ & $\overline{00}$ & 00 & 00 & 20 & 03 & 23 \\
\hline & $\begin{array}{c}\text { Gradua- } \\
\text { ção }\end{array}$ & 01 & 05 & 02 & 03 & 01 & 12 \\
\hline & $\begin{array}{l}\text { Especia- } \\
\text { lização }\end{array}$ & 10 & 00 & 04 & 00 & 00 & 14 \\
\hline & Mestrado & 01 & 00 & 00 & 00 & 00 & 01 \\
\hline
\end{tabular}

Fonte: Dados da pesquisa (2019)

Destaca-se neste quadro que a categoria de Enfermagem (Enfermeiros e Técnicos de Enfermagem), representa $70 \%$ dos profissionais que atuam nas Unidades Básicas de Saúde.

Para compreender a dinâmica de trabalho no que se refere à prática assistencial voltada ao atendimento dos casos de sífilis e ao perfil profissional dos trabalhadores pesquisados, buscou-se identificar as principais atividades desenvolvidas na atenção básica que teriam relação com a sífilis (quadro 2).

Quadro 2: Variáveis relativa às atividades dos profissionais da saúde da atenção básica participantes da pesquisa, Senhor do Bonfim, 2019.

\begin{tabular}{|l|c|c|}
\hline \multicolumn{1}{|c|}{ Atividade } & $\begin{array}{l}\text { Não tem } \\
\text { capacitação } \\
\text { para atender } \\
\text { portador de } \\
\text { sífilis* }\end{array}$ & $\begin{array}{l}\text { Não tem } \\
\text { experiências } \\
\text { em diagnosti- } \\
\text { Trabal e notificar } \\
\text { casos de sífilis }\end{array}$ \\
\hline Enfermeiros $(\mathrm{n}=12)$ & 02 & 01 \\
\hline Médicos $(\mathrm{n}=05)$ & 01 & 00 \\
\hline Odontólogos $(\mathrm{n}=06)$ & 03 & 04 \\
\hline $\begin{array}{l}\text { Técnico de enfermagem }(\mathrm{n}= \\
\text { 23) }\end{array}$ & 18 & 20 \\
\hline Recepcionistas $(\mathrm{n}=04)$ & 04 & 04 \\
\hline Total =50 & 28 & 29 \\
\hline
\end{tabular}

*Variáveis relacionadas à percepção do próprio entrevistado (autorreferida).

Fonte: Dados da pesquisa (2019). 
No que se refere à condição dos profissionais/trabalhadores de saúde, acerca das duas variáveis do quadro 02 (capacitação e experiência), observa-se que no tocante à capacitação 28 profissionais assumiram a inexistência da mesma e, em relação à experiência em diagnosticar e notificar, 29 deles afirmam não terem experiência, dentre estes, 36\% compõem a categoria dos técnicos de enfermagem. É imperativo salientar que $56 \%$ dos entrevistados afirmam dificuldades no atendimento.

Ao procedermos a Análise Fatorial de Correspondência (AFC) do corpus textual com auxílio do Iramuteq, obteve-se um gráfico (Figura 1). A AFC gerada possibilitou examinar as oposições e aproximações textuais acerca do pensamento dos profissionais/trabalhadores sobre notificação epidemiológica, diagnóstico e demais questões ligadas à sífilis. Como mostra a figura 1, cada classe textual se apresenta em cores diferentes (azul claro, azul escuro, cinza, rosa, verde e vermelho) e abrange contextos semânticos específicos que se referem à raiz semântica das palavras com maiores frequência e x2 que mais se expressaram em sua composição.

Figura - 1 Análise fatorial de correspondência, dos profissionais da saúde da atenção básica participantes da pesquisa, Senhor do Bonfim, 2019.

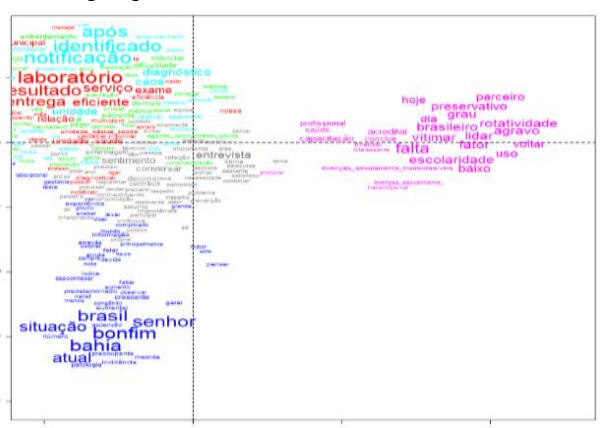

Fonte: Dados da pesquisa (2019).

Na AFC cada Classe foi denominada a partir dos elementos que a compõem. A Classe 1 apresenta em evidência palavras que remetem a exames e laboratório; a Classe 2, aponta elementos como sentimento e diálogo; a Classe 3 , reflete as dificuldade vivenciadas; a Classe 4, apresenta aspectos da notificação e diagnóstico; a Classe 5, a situação epidemiológica; e, a Classe 6 , aborda inquietações e limites operacionais.

A figura 1 possibilita, ainda, perceber a partição textual em expressões/palavras proferidas pelos profissionais/trabalhadores da saúde e analisá-las a partir de cada uma das seis classes que o compõe, da conexão entre as mesmas e da fragmentação do corpus em três eixos. O eixo I foi composto apenas pela classe 6 , apresenta-se em oposição a todas as outras e $25,6 \%$ do corpus textual a compõem; o eixo II apresenta as classes 1, 3 e 4 que de forma agregada alberga $46,0 \%$ do corpus; e, o eixo III com as classes 2 e 5 perfaz $28,4 \%$ do corpus. Observa-se, na figura 1, que a classe 6 tem maior proximidade com as classes 1,3 e 4 (eixo II), e se distancia das classes 2 e 5 (eixo III). Assim, para a análise da AFC, cada Eixo em seu arranjo, foi nomeado pelos autores e descritos conforme ordem de importância e elementos que a compõe.

\section{Eixo I - Classe 06 - Fatores ligados ao desempenho do profissio- nal/trabalhador na atenção básica no tocante a promoção e prevenção da sífilis}

Essa classe sinaliza que os profissionais/trabalhadores de saúde que atuam na UBS no atendimento ao portador de sífilis, de acordo com recorte das falas, se preocupam com as pessoas que se descuidam com sua saúde e revelam a falta de experiência profissional ao expressarem os termos 'capacitação e rotatividade':
Não recebi capacitação para atender o portador de sífilis, mas possuo experiência em diagnosticar e notificar casos de sífilis em unidade de saúde (M 15).

Tenho a impressão em relação as doenças sexualmente transmissíveis que as pessoas perderam o medo de adoecer e não fazem uso da prevenção como deveriam (E 1).

\section{Eixo II - Classes 1, 3 e 4 - Questões relacionadas à rede de serviços}

A Classe 1, no Eixo II, comporta elementos referentes a rede de laboratórios, própria e/ou conveniada ao SUS que oferta o exame, testes não treponêmicos (VDRL), para o diagnóstico da sífilis e, a secretaria municipal de saúde disponibiliza o Teste rápido em todas as UBS.

Os termos presentes na Classe 1 apontam que alguns profissionais/trabalhadores parecem demonstrar insatisfação com a prestação dos serviços ofertados, sobretudo, no que se refere a eficiência na entrega dos resultados. Para além, também evidenciam queixas quanto a demora e confiabilidade dos exames, conforme falas:

A relação da nossa unidade com o laboratório municipal no diagnóstico da sífilis, nem sempre temos resposta imediata por parte do laboratório, mas na minha área isso não é um grande problema, já que trabalho com uma população que possui plano de saúde em grande parte (E 5).

O laboratório não é eficiente na entrega dos exames e o resultado não são confiáveis, às vezes os resultados saem tudo errado, porque quando o paciente vai refazer no laboratório particular dá positivo (E 9).

Ainda com relação ao Eixo I, a Classe 3 apresenta dificuldades vivenciadas pelos profissionais no manejo 
da sífilis. Essa classe expõe elementos relativos aos enfrentamentos cotidianos em suas respectivas unidades de atuação. Nota-se que, no recorte das falas, a equipe não relata dificuldades no manejo dos pacientes com sífilis, o que eles referem, é que, às vezes, as dificuldades perpassam pela resistência/aceitação do diagnóstico e/ou abandono do tratamento.

Com relação às dificuldades ou enfrentamentos vivenciados por mim e pelos demais profissionais desta unidade no manejo da sífilis, no momento não temos dificuldade, medicação temos, médico tem também para prescrever, a nova norma técnica do Cofen, autoriza enfermeiros a prescrever e iniciar o tratamento, a gente não tem dificuldade. Às vezes a gente sente dificuldade de o paciente aderir ao tratamento. (E 1$)$

Ainda em relação as questões relativas a rede de serviços, a Classe 04 aponta elementos ligados diretamente ao pós-diagnóstico e notificação dos casos de sífilis. As palavras que compõem a Classe conotam aspectos que perpassam o conhecimento científico e estão relacionados à formação acadêmica e a vivência dos profissionais na atenção básica. Os excertos de fala demonstram que a equipe possui conhecimento técnico e científico embasados em protocolos clínicos e diretrizes terapêuticas para o tratamento da sífilis.

Após o diagnóstico e notificação dos caos de sífilis que foram identificados, nessa unidade o tratamento é feito com aplicação de Penicilina $G$ benzatina e a gente vai monitorando através do próprio paciente; a gente faz o agendamento para o tratamento e pede que o paciente venha durante três semanas para fazer a aplicação da Penicilina $G$ benzatina e con- versa para ver como é que está a reação dele e depois fica monitorando a cura, através do VDRL e quando o paciente se torna faltoso, a gente aciona o agente comunitário de saúde para fazer a busca ativa. (E 4).

Após o diagnóstico e notificação dos casos de sífilis é iniciado o tratamento do paciente aqui na unidade, a notificação é encaminhada para VIEP, para consolidar os dados e estabelecer o perfil epidemiológico para saber como está a situação de sífilis no município. (E 9)

\section{Eixo III - Classes 2 e 5 - Reporta aos sentimentos e conhecimento acerca da temática}

Na Classe 02 os elementos presentes manifestam a implicação dos profissionais na discussão da temática ao revelarem suas experiências e sentimento experenciado. Percebe-se uma consonância entre as falas e as políticas públicas de saúde, mas, manifestam sentimentos negativos no tocante à prescrição da medicação para tratamento dos pacientes com sífilis, conforme os recortes das falas a seguir:

O sentimento que tenho no decorrer dessa entrevista é de gratidão em poder explanar um pouco da minha vivência, com algo que ainda é tão alarmante no Brasil e perceber que existem pessoas, instituições de ensino engajadas nesse contexto e nos fazer acreditar na importância da saúde pública e da necessidade de continuar buscando e aprimorando nas políticas públicas de saúde. (E 2).

Em ralação a tudo que conversamos meu sentimento é de impotência diante dos casos de sífilis. Diagnostico e notifico, mas a situação é barrada na medicação que não tem, um resultado de exame que demora. (M 17).
O Eixo III comporta também a Classe 5, essa aborda a importância do profissional/trabalhador em saúde manter-se atualizado, conhecer a situação epidemiológica da sífilis e de outras IST.

A situação atual da sífilis, penso que está em ascensão muito grande, provavelmente pela falta da Penicilina $G$ benzatina. $E$ há uma necessidade de uma maior atenção no município, $e$ no Brasil houve uma ascensão há 5 meses atrás, por causa da falta da Penicilina G benzatina, a repercussão está vindo com a sífilis congênita. (M 13).

A situação atual da sífilis acho que a incidência ainda está grande, apesar das unidades ter os testes rápidos, principalmente no pré-natal, mas que ainda tem muitos paciente com incidência de sífilis, mesmo com os tratamentos a gente vê casos de sífilis congênita e no pré-natal é cobrado o tempo todo que deve se prevenir, diante disso poderia ter menos caos de sífilis. Apesar de campanhas contra sífilis e de conscientização das gestantes com relação à importância do pré-natal, ainda considera a sífilis uma patologia com incidência elevada e no município também. (O 50)

\section{DISCUSSÃO}

A permanência dos profissionais de saúde na ESF, em especial de médicos e enfermeiros, é considerada um dos fatores críticos para o sucesso da estratégia. A rotatividade dos mesmos pode comprometer a efetividade do modelo, prejudicando a qualidade da assistência e a satisfação dos usuários, que dependem do vínculo entre os profissionais da equipe e a população (GIOVANI; VIEIRA, 2013).

Em relação ao tempo de atuação dos profissionais que compuseram a amostra deste estudo (Quadro 1), percebe-se rotatividade de uma boa parte 
dos profissionais/trabalhadores no serviço público. A soma do porcentual entre os profissionais que atuam há menos de um ano e os que atuam entre 01 (um) e 05 (cinco) anos, totaliza $66 \%$ da amostra. Esse achado implica em comprometimento da qualidade da assistência decorrente do pouco tempo de atuação na atenção básica e aponta fragilidade nos vínculos estabelecidos com a comunidade.

Estudo de abordagem transversal, realizado com 811 profissionais vinculados às Equipes de Saúde da Família (ESF) em Montes Claros-MG, observou que a alternância de profissionais pode interferir na eficiência e eficácia da atenção à saúde, prejudicando a qualidade da assistência e a satisfação dos usuários, que dependem do estabelecimento de vínculo entre profissionais da equipe e população (TONELLI, et al., 2018). Assim, para alcançar êxito na assistência ofertada na atenção básica, é necessária uma equipe estável, que conheça a comunidade em sua dimensão sócio-políticocultural e adentre na sua história (MEDEIROS, et al., 2010).

Outro elemento observado no quadro $1 \mathrm{diz}$ respeito ao tempo de formação. Existe uma amplitude variando entre 01 (um) e 18 anos e $10 \%$ desses profissionais concluíram os estudos há menos de três anos. No entanto, a maior concentração (66\%) concluiu há mais de 10 anos, o que revela um tempo significativo de experiência profissional, do ponto de vista do conhecimento que pode ser adquirido ao longo dos anos.

Com base no quadro 2, o processo de trabalho na ESF se assenta no trabalho multidisciplinar. No entanto, pesquisa revela ser o enfermeiro um ator importante nas ações educativas, no fortalecimento do vínculo com a comunidade, na proximidade com a família e na articulação setorial, bem como implementar os cuidados de enfermagem em consonância com as diretrizes e os princípios do SUS (ABRAHÃO; SOUZA, 2013). Nesse sentido, vale ressaltar que os profissionais de enfermagem se apresentam em maior número, configurando $70 \%$ dentre os participantes deste estudo.

No entanto, as fragilidades das equipes da atenção básica apontam baixa capacitação e experiência. A Política da Atenção Primária, na tentativa de controlar ou erradicar os casos de sífilis, propõem elevar esses índices por meio contínuo de promoção de ações práticas e a redução do quadro nacional de subnotificação das sífilis ocasionado, principalmente, pela ausência do diagnóstico ou não tratamento adequadamente da sífilis (PINHEIRO, 2011). Por esta razão, salienta-se a necessidade de sensibilização, capacitação técnica e atitudinal de profissionais e gestores frente ao que preconiza os protocolos clínicos e recomendações institucionais à efetiva assistência no diagnóstico e tratamento da sífilis, conforme a existência de legislação específica, no que tange aos Conselhos Profissionais e MS (DOMINGUES; LEAL, 2016).

Cabe assinalar que, no município ora estudado, a atuação dos profissionais odontólogos no contexto de atenção à sífilis é parca, tanto no que tange capacitação quanto experiência em cuidar/atender esses usuários. Percebe-se, ainda, pouca interação destes profissional(ais) em questões que perpassem à saúde bucal ou haver pouco envolvimento destes no processo de atenção à sífilis, seja no diagnóstico e/ou no acompanhamento do paciente que apresenta tal patologia.

Dos 85 pacientes com sífilis adquirida estudados, com sinais observados na cavidade oral e que receberam atendimento médico em um Centro de Referência em Medicina Oral, em um hospital público brasileiro, as lesões orais poderiam ter representado uma suspeita diagnóstica caso os profissionais de saúde bucal fossem conscientizados e capacitados na tentativa de desenvolver a eficácia na suspeição clínica diagnóstica (MATIAS, et al., 2019). Portanto, ressalta-se a importância da interação do profissional odontólogo no processo de prevenção, diagnóstico e acompanhamento de pacientes com sífilis

Nesse estudo, observou-se que 29 participantes afirmaram não ter adquirido experiências em diagnosticar/notificar casos de sífilis em UBS. Tal confirmação, pode implicar, diretamente, na assistência oferecida aos usuários e comprometer sua saúde e até mesmo impactar em sequelas irreversíveis aos usuários dos serviços. Chama-nos atenção, em relação aos trabalhadores da saúde que, embora não apresentem conhecimento/experiências consolidadas para diagnosticar e notificar os casos de sífilis, continuam prestando assistência na UBS e podem incorrer em falhas no atendimento, subnotificação e/ou erros de diagnóstico. Diante disso, faz-se necessário que seja ofertada intervenção educacional para que esses profissionais adquiram qualificação técnica conforme preconiza o MS.

Estudo realizado no Paraná, no ano de 2017, evidenciou que houve mudanças e desenvolvimento relevante após intervenção educacional, ofertada aos profissionais de saúde, para realizar diagnóstico, notificação e manejo adequado do paciente com sífilis. Salienta-se que antes da intervenção os participantes da pesquisa não demonstravam conhecimento em conformidade com os parâmetros recomendado 
pelo MS (LAZARINI; BARBOSA, 2017).

Assim, é de suma importância a realização da avaliação clínica minuciosa, anamnese, exame físico e observar o aparecimento dos sinais e sintomas da sífilis por profissionais de saúde capacitados que atuam em UBS (BRASIL, 2018).

Com relação às análises oriundas das questões abertas, processadas pelo Iramuteq, a figura 1 apresenta em seu Eixo I, na Classe 06, fatores ligados ao desempenho do profissional/trabalhador na atenção básica no tocante à promoção e prevenção da sífilis. Isto pode ser associado aos achados do estudo (PINHEIRO, 2011), que utilizou dados de 2011/2012 de hospitais e de 23.894 puérperas, e concluiu que os casos de sífilis congênita estavam imbricados na baixa escolaridade materna, início mais tardio do pré-natal, menor número de consultas e menor realização de exames sorológicos. Confirmando que o baixo desempenho dos profissionais influencia negativamente na promoção da saúde, prevenção de doenças e tratamento necessário.

Com relação à notificações de sífilis em gestantes, estudo realizado em quatro municípios do Rio Grande do Sul, com participação de 17 profissionais, concluiu que a composição das equipes e a formação das redes assistenciais requer práticas pedagógicas e educação permanente para dirimir obstáculos como a rotatividade de profissionais e a centralização de ações no enfermeiro, a fim de fortalecer as ações das equipes multiprofissionais frente a abordagem da sífilis (BAGATINI, et al, 2018).

Nesse estudo, no Eixo II composto pelas Classes 1, 3 e 4, as questões ligadas à rede de serviços revelaram que a relação do laboratório muni- cipal com as UBS necessita ser ajustada, com vista a atender as demandas solicitadas em tempo hábil, garantir a confiabilidade dos resultados dos exames e não comprometer a assistência prestada aos usurários com suspeita de sífilis. Frente a essa delicada situação pode-se inferir que o serviço prestado pelo laboratório impõe dificuldades para que a atenção primária se aproxime do que preconiza o MS. A não confiabilidade no resultados dos exames realizados configura como um fator de risco para os usuários. Nesse sentido, um participante relatou que os resultados dos exames não são confiáveis e que com certa frequência, recebiam exames com resultado falsonegativo.

Os Laboratórios integram a rede local de serviços para a realização de exames básicos e essenciais. São estruturas unitárias que possuem a atribuição de atender às demandas oriundas das necessidades mais comuns da comunidade, buscando a resolubilidade dentro do seu nível de competência. Um diagnóstico laboratorial preciso é uma excelente ferramenta na precaução e/ou confirmação da doença e assim dar início ao tratamento (SANTOS, 1997). Ressalta-se que a triagem laboratorial da sífilis "tem baixo custo e não requer laboratórios sofisticados ou amplamente equipados". Depende, principalmente, da existência de uma rede de saúde bem estruturada e imbuída na redução da prevalência desta doença (FIRMO, et al., 2013).

Um estudo exploratório e descritivo, por meio de dados secundários coletados nas fichas de investigação/notificação de sífilis, com mulheres assistidas em Hospital Universitário Materno Infantil, identificou dificuldade e resistência dos profissionais de saúde para realizar o tratamento do parceiro sexual de portadores de IST.
Tal achado coaduna com a dificuldade histórica de implementação da política pública de saúde do homem e sua baixa adesão nas UBS, visto que não ter acesso ao parceiro dificulta o tratamento adequado e a quebra da cadeia de transmissão (DANTAS, et al., 2017). Em Uganda, país da África Oriental, o estudo denominado Tratamento de Sífilis de Parceiros (STOP) concluiu que a dificuldade da adesão masculina perpassa ao conhecimento limitado sobre sífilis, medo de injeção dolorosa, falta de habilidades de comunicação e cultura masculina (NAKKU-JOLOBA, et al., 2019).

No Brasil, de acordo com os dados atuais do SINAN, os números de casos de sífilis, tanto de morbidade quanto de mortalidades, são inquietantes. Já foram implantadas diversas políticas de controle de prevenção dessa patologia, exigindo dos profissionais de saúde aplicação de conhecimento técnico e científico adequados no atendimento às pessoas contaminadas e/ou com suspeita de sífilis, na tentativa de reverter esse quadro e ofertando assistência de qualidade.

O presente estudo conota, por meio das falas, que a maioria dos médicos e enfermeiros expressaram conhecimentos técnicos e científicos, habilidades e ações relacionadas ao pós-diagnóstico e notificação, sendo essa embasadas em protocolos clínicos e diretrizes terapêuticas para o tratamento da sífilis, como consta nos excertos apresentados na Classe 4 do eixo II. Demonstrando estarem em ressonância com as normas estabelecidas pelo MS e nota técnica do Conselho Federal de Enfermagem - COFEN/CTLN No 03/2017 que asseguram aos médicos e enfermeiros, no âmbito das UBS, a prescrição da Penicilina G Benzatina. 
Nesse sentido, os enfermeiros, em suas falas, demonstram embasamento ancorado na nota técnica e realizavam a prescrição da medicação para o tratamento da sífilis reafirmando, assim, o compromisso com a saúde dos usuários da UBS. Porém, uma parcela dentre os profissionais com ensino superior, que têm a atribuição de diagnosticar e notificar casos de sífilis, demonstrou possuir parcial conhecimento com relação à temática. Tal achado implica negativamente no processo de redução da epidemia e fragiliza políticas de controle e prevenção da doença.

Nesse sentido, estudo realizado em Fortaleza-CE-Brasil, que utilizou questionário auto aplicado e considerou como respostas corretas aquelas que estivessem em conformidade com as recomendações do MS, concluiu que os servidores não possuíam conhecimento adequado acerca das ações preventivas e do controle da sífilis congênita (SILVA, et al., 2014). Estudo que aborda uma outra doença negligenciada, a hanseníase, também aponta a dificuldade dos profissionais em lidar com ações de prevenção (COSTA; SANTOS; ALVES, 2020).

O Eixo III, composto pelas Classes 2 e 5, reporta sentimentos antagônicos e conhecimento acerca da situação atual da sífilis. Um dos recortes da fala, presente na Classe 5, relembra que nos últimos anos o país experimentou temporariamente um desabastecimento nacional de Penicilina $G$ Benzantina, em virtude da ausência de matéria prima para produção no mercado mundial, o que ocasionou em aumento de casos da doença.

Diante da temática discutida e fazendo referência a situação atual da sífilis, estudo realizado em um município de médio porte populacional em São Paulo apresenta achados seme- lhantes e aponta que o aumento dos casos de sífilis podem estar associados ao baixo grau de escolaridade, diminuição do uso do preservativo, rotatividade de parceiros e tratamento não realizado ou inadequado, para além das questões relacionadas aos profissionais e de gerenciamento (LINO; MOTA; SOUSA, 2019).

\section{CONSIDERAÇÕES FINAIS}

Os achados deste estudo revelaram que a falta de capacitação e experiência dos profissionais/trabalhadores em saúde, apesar de atuarem na $\mathrm{AB}$ entre um e cinco anos, no que se refere ao diagnóstico e notificação da sífilis se apresenta fragilizado. Revelaram, ainda, que os odontólogos e técnicos de enfermagem, apesar do respaldo legal, não desenvolvem ações relativas à realização do teste rápido e notificação de casos.

As fragilidades apontadas pelos participantes do estudo, quanto à assistência prestada a pessoa com suspeita de sífilis e às deficiência nas ações ofertadas, perpassam desde o diagnóstico até a notificação e tratamento. Desvelam necessidades de capacitação da equipe de saúde e provimento de insumos como limites para o enfrentamento da epidemia. Os achados apontaram, ainda, para a necessidade de promoção de estratégias eficazes, como a do aconselhamento para atendimento às vulnerabilidades de usuários e indicam que intervenções políticas, gerenciais e de educação em serviço, direcionadas às equipes, necessitam vir a ser implantadas para o manejo eficaz da sífilis.

Considera-se como limitação do estudo a não ampliação do número de unidades básicas de saúde devido a opção em incluir apenas as unidades localizadas na sede do município.
Além disso, um recorte que envolvesse profissionais que atuam em serviços de média complexidade ou municípios de maior porte, talvez, pudesse evidenciar outros elementos para análise, portanto, sugere-se novos estudos que venham complementar essas informações.

\section{REFERÊNCIAS}

ABRAHÃO, A.L.; SOUZA, R.F. O trabalho do enfermeiro na estratégia saúde da família-aspectos da prática promocional em saúde. Rev da rede Enferm do N.E. v.14, n. 4, p. 740747, 2013. Disponível em: http://www.repositorio.ufc.br/handle/ri ufc/11558

BAHIA. Secretaria da saúde. SuvisaDivep. Informe Epidemiológico de Sífilis. p. 1-8, 2019. Disponível em: http://www.saude.ba.gov.br/suvisa/bol etinsepidemiologicos

BAGATINI, C. L. T; MACHADO, R. Z.; CECCIM, R. B; BAVARESCO, C.S. Teste rápido para sífilis no prénatal da atenção básica: avaliação institucional qualitativa e educação permanente em saúde. Saúde em Redes. v. 2, n. 1, p. 81-95, 2018. Disponível em:

https://doi.org/10.18310/24464813.2016v2n1p81-95

BRASIL. Ministério da saúde. Secretaria de Vigilância em Saúde. Boletim epidemiológico de sífilis. Ministério da saúde, 2019. Disponível em: http://www.aids.gov.br/ptbr/pub/2018/boletim-epidemiologicode-sifilis-2018

. Ministério da saúde. Secretaria de Vigilância em Saúde. Departamento de DST, Aids e Hepatites Virais. Protocolo Clínico e Diretrizes Terapêuticas para prevenção da transmissão vertical de HIV, Sífilis e Hepatites Virais. Brasília, DF: Ministério da saúde, 2018. Disponível em: http://www.aids.gov.br/ptbr/pub/2015/protocolo-clinico-ediretrizes-terapeuticas-para-prevencao- 
da-transmissao-vertical-de-hiv

CAMARGO, B.V.; JUSTO, A.M. Iramuteq: um software gratuito para análise de dados textuais. Temas em psicologia, v. 21, n. 2, p. 513-518, 2013. Disponível em: http://dx.doi.org/10.9788/TP2013.2-16

COSTA, A. K. A. N.; SANTOS, Á. N.; ALVES, K. A. N. Tendência da hanseníase em menores de 15 anos por região do Brasil entre 2007 a 2017.

Rev. ComCiência, v. 6, n. 1, p. 22-25, 2020. Disponível em:

http://www.revistacomciencia.com/arq uivos/35.pdf

DANTAS, L. A.; JERÔNIMO, S. H. N. M.; TEIXEIRA, G. A.; LOPES, T. R. G.; CASSIANO, A. N.; CARVALHO, J. B. L. Perfil epidemiológico de sífilis adquirida diagnosticada y notificada en hospital universitario materno infantil. Enfem global, v. 16, n. 2, p. 217-245, 2017. Disponível em: http://dx.doi.org/10.6018/eglobal.16.2. 229371

FIRMO, W. C. A.; PAREDES, A. O.; ALMEIDA, A. C.; CAMPOS, M. C.; PIMENTEL, M. I. Z.; PONTES, S. R. S. Perfil dos exames laboratoriais em gestantes atendidas no Centro de Saúde de Lago Verde. Journal of Manag Prim Health Care, v. 4, n. 2, p. 7786, 2013. Disponível em:

http://jmphc.com.br/jmphc/article/view $/ 173$

GIOVANI, M. S. P.; VIEIRA, C. M. Longitudinalidade do cuidado diante da rotatividade de profissionais na Estratégia Saúde da Família. Rev eletr de Com Inf Inov Saúde, v.7, n. 4, p. 1-14, 2013. Disponível em: http://dx.doi.org/10.3395/reciis.v7i4.5 72.

LAZARINI, F. M.; BARBOSA, D. A. Intervenção educacional na Atenção Básica para prevenção da sífilis congênita. Rev Lat Am Enferm, v. 25, e2845, 2017. Disponível em: https://www.redalyc.org/articulo.oa

LINO, C. M.; MOTA, M. J. B. B.; SOUSA, M. L. R. Sífilis adquirida, em gestante e congênita: perfil epide- miológico em um município de médio porte do Estado de São Paulo. [São Paulo]: Universidade Estadual de Campinas, 2019. Disponível em: http://repositorio.unicamp.br/jspui/han dle/REPOSIP/334067

LOPES, A. C. M. U.; ARAUJO, M. A.L.; VASCONCELOS, L. D. P. G.; UCHOA, F. S. V.; ROCHA, H. P.; SANTOS, J. R. Implantação dos testes rápidos para sífilis e HIV na rotina do pré-natal em Fortaleza- Ceará. Rev Bras Enferm, v. 69, n. 1, p. 62-66, 2016. Disponível em:

http://dx.doi.org/10.1590/00347167.2016690108i

MATIAS, M. D. P.; JESUS, A. O.; RESENDE, R. G.; CALDEIRA, P. C.; AGUIAR, M. C. F. Diagnosing acquired syphilis through oral lesions: the 12 year experience of na Oral Medicie Center. Brasilian Journal of Otorhinolaryngology, p. 1-6, 2019. Disponível em:

https://doi.org/10.1016/j.bjorl.2018.12. 010

MEDEIROS, C. R. G.; JUNQUEIRA, A. G. W.; SCHWINGEL, G.; CARRENO, I.; JUNGLES, L. A. P.; SALDANHA, O. M. F. L. A rotatividade de enfermeiros e médicos: um impasse na implementação da Estratégia de Saúde da Família. Ciênc saúde colet, v. 15 , n. 1, p. 1521-1531, 2010. Disponível em:

http://dx.doi.org/10.1590/S1413-

81232010000700064

MENDES, F. R. P.; ZANGÃO, M. O. B.; GEMITO, M. L. G. P.; SERRA, I. C. C. Representações sociais dos estudantes de enfermagem sobre assistência hospitalar e atenção primária. Revi Bras Enferm, v. 69, n. 2, p. 345-350, 2016. Disponível em: http://dx.doi.org/10.1590/00347167.2016690218i

MINAYO, M. C. S. Pesquisa social: teoria, método e criatividade. Petrópolis, RJ: Vozes, 2016 (Série Manuais Acadêmicos). 95p.
MIZEVSKI, V. D.; BRAND, E. M.; CALVO, K. S.; BELLINI, F. M.; MACHADO, V. S.; DUARTE, E. R. $\mathrm{M}$, et al. Disponibilidade do teste rápido para sífilis e anti-HIV nas unidades de atenção básica do Brasil. Saúde em Redes, v. 3, n. 1, p, 40-49, 2017. Disponível em: https://doi.org/10.18310/24464813.2017v3n1p40-49

NAKKU-JOLOBA, E.; KIGULI, J.; KAYEMBA, C. N.; TWIMUKYE, A.; MBAZIRA, J. K.; PARKES-

RATANSHI, R.; BIRUNGI, M.; KYENKYA, J.; BYAMUGISHA, J.; GAYDOS, C.; MANABE, Y. C. Perspectives on male partner notification and treatment for syphilis among antenatal women and their partners in Kampala and Wakiso districts, Uganda. BMC Infect Dis. v. 19, n. 1, p. 124, 2019. Disponível em: 10.1186/s12879-019-3695-y

PINHEIRO, V. A. O. Aspectos científicos, epidemiológicos, preventivos, diagnóstico e de tratamento relativo à sífilis e sífilis congênita no Brasil: uma revisão bibliográfica [monografia]. Minas Gerais: Universidade Federal de Minas Gerais, 2011. Disponível em:

https://www.nescon.medicina.ufmg.br/

SANTOS, A. B.; VILHENA, M. A. H.; MAIA, M. T.; CAVALCANTE, M. N. P.; BAIOCCHI, B. P. Sífilis Congênita: Uma análise temporal da incidência no município de Teresópolis/RJ. Anais da IV Jornada de pesquisa e iniciação científica do UNIFESO: Ciência e desenvolvimento social, 2014. Rio de Janeiro, Brasil. Disponível em:

http://www.unifeso.edu.br/editora/pdf/ anais1.pdf

SILVA, D. M. A.; ARAÚJO, M. A. L.; SILVA, R. M.; ANDRADE, R. F. V.; MOURA, H. J.; ESTEVES, A. B. B. Knowledge of healthcare professionals regarding the vertical transmission of syphilis in Fortaleza-CE, Brazil. Texto contexto, v. 23, n. 2, p. 275 85, 2014. Disponível em: http://dx.doi.org/10.1590/010407072014000510013 
TIAGO, Z. S.; PICOLI, R. P.; GRAEFF, S. V. B.; CUNHA, R. V.;

ARANTES, R. Subnotificação de sífilis em gestantes, congênita e adquirida entre povos indígenas em Mato Grosso do Sul. Epidemiol Serv Saúde, v. 26, n. 3, p. 503-512, 2017. Disponível em:

http://dx.doi.org/10.5123/s1679-

49742017000300008

TONELLI, B.; LEAL, A. P.; TONELLI, W.; VELOSO, D. C.; GONÇALVES, D.; TONELLI, S. Rotatividade de profissionais da Estratégia Saúde da Família no município de Montes Claros, Minas Gerais, Brasil. Rev Facul Odonto-UPF, v. 23, n. 2, p. 180-185, 2018. Disponível em:

https://doi.org/10.5335/rfo.v23i2.8314 